\title{
The Role of Histone Deacetylase and Histone Deacetylase Inhibitors in Rheumatoid Arthritis
}

\section{Rina Kriswiastiny ${ }^{1 *}$, Radiyati Umi Partan ${ }^{1}$, Hermansyah¹, Surya Darma ${ }^{1}$, Muhammad Reagan ${ }^{1}$}

${ }^{1}$ Rheumatology Subdivision, Department of Internal Medicine, Faculty of Medicine, Universitas Sriwijaya, Palembang, Indonesia

\section{A R T I C L E I N F O}

\section{Keywords:}

Rheumatoid arthritis

HDAC

HDACi

Anti-inflammatory

\section{*Corresponding author:}

Rina Kriswiastiny

\section{E-mail address:}

\section{rinakriswiastiny@yahoo.com}

All authors have reviewed and approved the final version of the manuscript.

https://doi.org/10.32539/bsm.v5i11.423

\begin{abstract}
A B S T R A C T
Rheumatoid Arthritis or RA disease is a chronic inflammatory and systemic disease associated with broad synovitis resulting in erosion of the articular cartilage and marginal bone causing joint damage. RA is an autoimmune disease with the discovery of an autoantibody, namely rheumatoid factor. The relevant antibody is anti-citrullinated protein (ACPA) antibody. Citullination on the introduction of several proteins such as fibrin, vimentin, fibronectin, collagen type II, which is expressed in the synovial membrane during inflammation by ACPA. In a recent study increased HDAC activity in the synovial tissue of RA patients has increased. Histone deacetylase or abbreviated as HDAC is an enzyme that is important in regulating gene transcription by altering the acetylation of histone proteins which results in an important regulation of repression in the implementation of inflammation. HDAC enzyme inhibitor (HDACi) is an HDAC enzyme inhibitor that can provide benefits for the treatment of various diseases including malignancy and inflammation. In inflammatory disease HDACi overcomes inflammatory cytokines such as TNF-a, IL6 and IL-1 $\beta$. One of the HDAC classes is HDAC1 which is highly expressed in the synovial fibroblasts of RA patients. HDACi can burden swollen joints, reduce mononuclear cell infiltration, request pannus orders, inhibit bone and cartilage damage.
\end{abstract}

\section{Introduction}

Rheumatoid Arthritis (RA) is an inflammatory arthritis disease that affects $0.5 \%-1 \%$ of the world's population. In addition to the joints, RA manifests in extra-articular organs such as the heart, lungs, vasculitis, nodules and can lead to the formation of atherosclerosis. RA is systematic because it can involve multiple organs, progressive disability, and even death. Advances in understanding the pathogenesis of RA have led to better management. However, current conventional and biological modification therapies sometimes fail and have not achieved the desired remission. This requires continuous therapy. The high mortality rate in RA patients and other cardiovascular and systemic complications is a challenge to find a better therapy. Although the exact cause is still unknown, environmental and genetic factors are involved in RA. In RA, the synovium can turn into invasive hyperplastic tissue that can cause cartilage and bone damage and even tendons and ligaments. Damage to cartilage and articular bone can be irreversible soon after the onset of RA. Fibroblast-like synoviocytes (FLS), which form the lining of the joints, in RA epigenetically have a more aggressive phenotype. FLS produce extracellular matrix and joint lubricants and produce mediators of cytokines and proteases that play a role in the pathogenesis of RA. Early treatment can improve the long-term improvement of RA patients. ${ }^{1.2}$ The concept of epigenetics was developed 
more than 70 years ago by Waddington in 1939. This theory develops in the theory of chromosomal inheritance, determining the structural details of genetic material and the mechanisms that mediate such inheritance and architecture such as modification of deoxyribonucleic acid (DNA) and histones. Epigenetic changes such as DNA methylation, histone modifications, and noncoding RNA, are thought to be involved in the pathogenesis of RA. Epigenetic modifications may influence $\mathrm{RA}$ via fibroblast-like synoviocytes (FLS). FLS has an important role in the onset and exacerbation of RA, so FLS plays a role in the pathogenesis of RA. In RA FLS there is an imbalance between histone acetyltransferase and histone deacetylase. ${ }^{3.4}$ The initiation of RA can begin several years before the onset of clinical symptoms. Disease onset involves specific genes that play a role in the production of pathogenic antibodies that bind to proteins and play a role in autoreactivity. The initial phase is characterized by repeated exposure to environmental stressors such as smoking, bacteria, viruses, and other environmental stimuli that can contribute to the immune response. 1 From several studies, patients with RA newly diagnosed in 3 months can experience erosion by $25 \%$ and $70 \%$ in 3 years. Delaying RA treatment for more than 90 days will cause more damage and require longer treatment. Early treatment of RA can reduce the radiological progression of joint damage by $33 \%$ compared to treatment of advanced RA patients. ${ }^{178,179}$. In addition, from several studies, remission of RA patients on monotherapy or combination therapy is still low. For example in the IDEA study, comparing remission on MTX monotherapy with MTX+Infliximab found remission of $50.8 \%$ and $40.6 \%$, respectively. Then in the AVERT study, $45.2 \%$ remission was obtained for MTX and $60.9 \%$ for MTX+Abatacept with a disease duration of less than 1 year. In addition, in RA patients who have suffered from RA for more than 1 year, the remission rate is also low. For example, in the ASPIRE study, MTX+infliximab remission was $21.20 \%$ and MTX alone was $15.00 \%$. The RA study began, barticinib+MTX therapy achieved remission of $40.00 \%$ while MTX alone was $24.00 \%$. Thus, the achievement of remission for the treatment of bDMARDs, tsDMARD, and csDMARD alone or in combination is still below $60 \%$. Therefore, other therapies are needed that may be more specific to the joint tissue itself so that it is expected to get a better remission. ${ }^{5-8}$

From the above review, it was found that FLS (fibroblast-like synoviocytes) in RA plays a very important role in the progression and destruction of bone in RA joints. This FLS has a semi-transformed, auto-reactive phenotype characterized by loss of inhibition, reduced apoptosis, and production of matrix-degrading enzymes. This trait is due in part to changes in epigenetic regulation associated with the repression of DNA transcription. DNA transcription repression is caused by reduced acetylation of amino acids in the tails of histone proteins which is an epigenetic sign. Repression of gene transcription, regulated by an enzyme called HDAC (histone deacetylase). Several studies in animal and human models of RA have shown the ability to reduce inflammation and joint tissue damage with the administration of HDAC inhibitors. 9 Thus HDAC is a new therapeutic target that can be a treatment option in RA patients. So far, RA treatment includes nonsteroidal anti-inflammatory drugs (NSAIDs), csDMARD (conventional disease-modifying anti-rheumatic drugs), biologic DMARDs, and specific target DMARDs and glucocorticoids. Treatment of bDMARD and tsDMARD is a drug that is used for a long time and is expensive. Discontinuation of the drug can also cause disease activity to worsen again. Recently, a treatment target was found to prevent early joint erosion, prevent synovial tissue hyperplasia, reduce swelling and reduce proinflammatory cytokines, namely HDACi. HDACi is a histone deacetylase inhibitor, an enzyme that can suppress transcription, replication, and repair of a gene. Examples of HDACi that have been developed include the inhibitor pan HDAC, ITF 2357, and SAHA (Suberoylanilide hydroxamic acid) which inhibit all HDACs. Selective HDACi such as Tubastatin A and Tubacin specifically inhibit HDAC6. While HDAC3 regulates STAT 1 activity in FLS RA. New HDAC 6 specific CKD-L. CKD-L can increase CTLA-4 in Fop3+ T cells and inhibit $\mathrm{T}$ cell proliferation. CDL can increase 
IL-10 and inhibit TNF-a and IL-1 $\beta .10$

\section{HDAC}

In the management of RA, there are still many remissions that have not been achieved or remissions that do not last and still need further treatment. Current treatment with biologic DMARDs and tsDMARDs is expensive. With the development of the management of RA, recently looked at the effect of epigenetic changes in Fibroblast synoviocytes (FLS) that cause chronic inflammation. Epigenetics is the origin of the Greek word epi- which means above, near, on, before, or after. But the words before or after may be used more often. Epigenetics can be defined as changes in the regulation of gene expression that can be passed on to progeny cells without changes in the nucleotide sequence of genes. Or the epigenetic mechanism is a mechanism that changes the pattern of gene expression and cellular function without changing the DNA sequence. Epigenetic mechanisms can theoretically play a role in 3 aspects of RA, namely the etiology of the disease, the mechanism of disease development, as a strong biomarker of disease development and activity. Monozygotic and dizygotic twin patients showed a more significant role of epigenetics than genetics in RA susceptibility. Histone deacetylase (HDAC) inhibitors are HDAC inhibitors that can suppress HDAC have been widely studied. One of them is an HDAC inhibitor that suppresses the production of IL-6 in RA-FLS. Pan HDACs such as ITF 2357 and SAHA inhibits all HDACs, while Tubastin A and Tubacin are HDAC inhibitors that selectively inhibit HDAC6. HDAC3 regulates STAT activity in RA-
FLS, whereas HDAC3 could be a potential therapeutic target in RA. HDAC6 inhibitor, CKD-L can play a role in improving the degree of arthritis. CKD-L increases CTLA-4 in cells and the Foxp3 inhibits $\mathrm{T}$ cell proliferation, increased IL-10 inhibits TNF-a, and IL$1 \beta$. How and the role of HDAC in RA will be discussed further. ${ }^{11-13}$

Histones are small, alkaline, and positively charged proteins, which allow them to be closely associated with DNA. Histones have globular domains, fold and tail domains at their amino and carboxyl ends whose residues undergo post-translational enzymatic modification. Histone protein modification in DNA is an epigenetic process in the regulation of DNA structure and function. Genetic modification plays a role in chromatin remodeling, changes in gene expression, and changes in cell phenotype types. The modifications that occur in histones vary widely. Many modifications occur in the $\mathrm{N}$-terminal or $\mathrm{C}$-terminal tails of these histones. This tail extends beyond the histone domain where the DNA is wrapped. Approximately 60 histone modifications have been studied. However, the most common are acetylation, methylation, phosphorylation, ubiquitylation, sumoylation, ADP ribosylation, deimination, proline isomerization, crotonylation, propylation, butyrylation, formylation, hydroxylation, and O-Glcnacylation. Compared to methylation and phosphorylation, acetylation is the most widely understood and studied. ${ }^{7}$ Histone acetylation occurs when the amino group of the converted lysine residue is located at the $\mathrm{N}$ terminal. All of these histones are acetylated in vivo. In this paper, we only discuss HDACassociated histone acetylation in RA patients. ${ }^{15-17}$

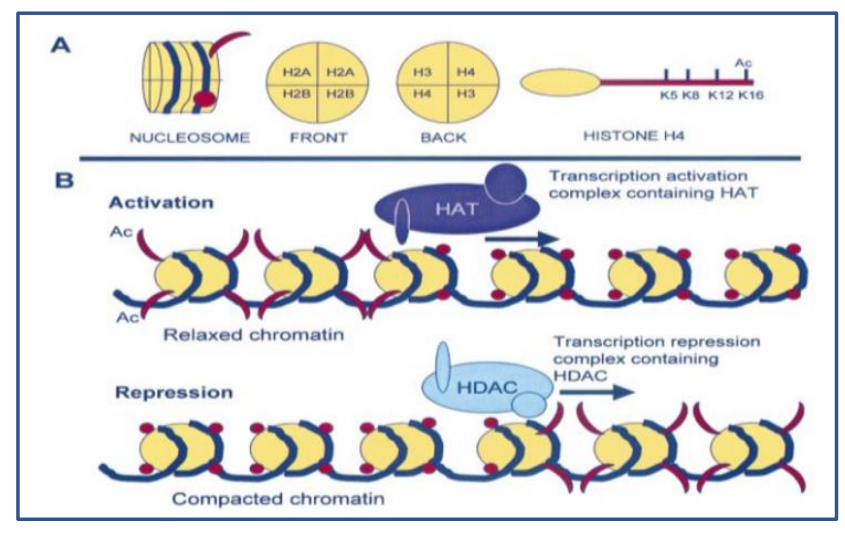

Figure 1. Various aspects of transcriptional and regulatory processes with histone modifications. ${ }^{18}$ 
Hyperacetylation can lead to increased expression of certain genes and hypoacetylation vice versa. Histone deacetylases (HDACs) play a role in removing acetyl groups from histones, to influence the expression of genes encoded by DNA in histone molecules. In addition to its role in histone modification, HDAC also removes acetyl groups from lysine residues in various other proteins including a transcription factor called lysine deacetylase. Thus, its effect on cellular processes is more complex and extensive than that of histone modification alone. Histones in their work are regulated by enzymes. These histone-modifying enzymes can add or remove these covalent modifications. These changes can affect the interaction between DNA and histones which will play a role in the ability of DNA to be transcribed. Enzymes that play a role in modifying histone residues also have non-histone substrates such as transcription factors. Two mechanisms for histone modification function are relaxation/unraveling or chromatin compression and recruitment of non-histone proteins. It depends on the type of modification and the amino acid residues involved. These histone modifications can lead to gene activation or repression. For example, the addition of an acetyl group to the histone tail of H3 makes lysine basic charge neutral and causes chromatin to open so that transcription occurs. Conversely, if the acetyl group is removed it will result in chromatin compression and prevent transcription. In transcription, DNA replication and repair each require a different set of modifications and require the enzymes involved. 19

Epigenetic regulators can be divided according to their function: 1. Epigenetic writers put their mark on DNA or histones. 2. Epigenetic erasers, marks on DNA or histones are removed by epigenetic erasers. 3 . Epigenetic readers, recognized by epigenetic readers. Histone modifications $\mathrm{H} 3$ and $\mathrm{H} 4$ are more extensive than H2A and H2B. Acetylation sites Lys ${ }^{9}$ and Lys ${ }^{14}$ on histones H3 and Lys ${ }^{5}$, Lys ${ }^{8}$, Lys ${ }^{12}$, Lys ${ }^{16}$ on H4. The stability of histone core acetylation is derived from the balance of opposing activities of histone acetyltransferases and histone deacetylases. Increased levels of histone acetylation (hyperacetylation) are associated with increased transcription and decreased levels of acetylation (hypoacetylation) are associated with repression of gene expression. 33,101,105,110,111,112, 123 Epigenetic writers such as histone acetyltransferases (HATs), histone methyltransferases (HMTs), protein arginine methyltransferases (PRMTs), and kinases put epigenetic markers on amino acid residues in histone tails. Epigenetic readers such as proteins containing bromodomains, chromodomains, and Tudor domains bind to these epigenetic markers. Epigenetic erasers such as histone deacetylases (HDACs), lysine demethylases (KDMs), and phosphatases catalyze epigenetic marker erasure. The addition and deletion of these post-translational histone tail modifications lead to the addition and/or deletion of other marks in the highly complex histone code. Histone modification regulates the processes of transcription, replication, and DNA repair. ${ }^{20}$

Histone acetylation plays a role in the regulation of gene expression and is associated with a dense region of transcriptionally active genes called euchromatin. Histone acetylation can be transient and can be maintained by enzymatic activity. Histone acetyltransferases (HATs) transfer the acetyl group (COCH3) from acetyl coenzyme A to lysine residues and neutralize the positive charge of lysine causing loose interactions between DNA and histones. These changes in chromatin structure lead to the accessibility of DNA to transcription factors and ultimately gene expression to occur. Whereas histone deacetylation catalyzed by histone deacetylases (HDACs) removing acetyl groups (deacetylation of lysine residues) causes a close interaction between negatively charged DNA and positively charged histones, These changes in chromatin structure make DNA inaccessible to transcription factors and ultimately the expression of these genes is suppressed. HAT enzymes such as CREB/p300, GCN5, MOF and PCAF are involved in gene regulation. While HDAC has 18 enzymes consisting of 4 classes. HAT and HDAC can modify various non-histone proteins with their activity depending on their acetylation statuses such as 
transcription factors, chaperone proteins, signal transduction mediators, and inflammatory mediators. Changes in acetylation status are related to protein stability, protein-protein interactions and protein-DNA interactions. HAT can modify more than one lysine residue but its specificity is limited. The acetyl group is added to the -N-terminal "histone tail" which makes it easy to access for modification. Reversal of acetylation by HDAC correlates with transcriptional repression. HDAC regulates cellular function, cell cycle progression, cell survival and proliferation. ${ }^{21}$

HDAC Enzyme Action Mechanism. The mechanism of action of the HDAC enzyme involves the removal of acetyl groups from histones that make up nucleosomes. This transfer results in hypoacetylation in the decrease in the space between the nucleosome and the DNA that surrounds it. This denser DNA encapsulation reduces the accessibility for transcription factors resulting in transcriptional repression. The HDAC catalytic domain is formed by a C390 amino acid stretch consisting of a single amino acid group. The active site consists of a curved tubular pouch with a wider base. The removal of the acetyl group occurs via a charge relay system consisting of two adjacent histidine residues, two aspartic residues (located about 30 amino acids from histidine and separated by about 6 amino acids), and one tyrosine residue (located about 123 amino acids downstream of the residue). aspartic). An important component of the relay system is the presence of $\mathrm{Znions}^{2+}$. These atoms are bonded to a zinc-binding site at the bottom of the pouch. Other cofactors are required for HDAC activity. The function of HDACi is to displace zinc ions and render the relay system inoperative. TSA (Trichostatin A) with its hydroxamic acid group and its five-carbon linkage to the phenyl group, has an optimal conformation to suit the active site. TSA is the most powerful reversible HDACi. All HDACs were equally sensitive to inhibition by TSA. 23

\section{Expression and activity of HDAC and HDACi in RA patients}

In RA patients there is an irregularity in the expression and activity of HDAC members. In a study in RA FLS in RA synovial tissue extract cores, an increase in HDAC activity were correlated with elevated levels of TNF-a. In addition, from RNA sequencing data from synovial biopsies, there was an increase in HDAC1 in RA patients who were not treated earlier. Meanwhile, another study analyzed synovial tissue extracts in which the total HDAC expression activity was upregulated and the expression of HDAC1 and HDAC2 decreased. Class III family members SIRTUIN, SIRT1 and SIRT2 are also regulated in RA FLS. The difference in the results of the study of RA patients in synovial tissue and PBMC was due to the small number of samples, different stages of disease and treatment, the presence or absence of controls that matched age and sex as well as different isolation where HDAC activity could change rapidly. In addition, PBMCs are heterogeneous cells and only a small part of them are likely to be involved in the pathogenesis of autoimmune diseases, resulting in varying results depending on the general state of the patient, 24

The specific deletion of HDAC $1 \mathrm{~T}$ cells in mice with RA inhibits CIA development. The reduced HDAC1 in T cells also caused the inflammatory cytokines IL-17 and IL-6 to significantly decrease in mouse serum. HDACs removing members in a particular cell lineage is not always beneficial for RA, which SIRT1 deficiency in myeloid cells can cause more severe arthritis, the number of osteoclasts and macrophages increased with an increase in IL-1 $\beta$ and TNF-a in joint tissue. overexpression of SIRT6 in intra-articular cells, resulting in decreased disease severity and cytokine production in joint tissue. ${ }^{25}$

HDACi potentially has antiproliferative effects that can stop the proliferation of aberrant synovial fibroblasts. In a study that analyzed the therapeutic effects of HDACi, class I HDACi reduced disease, inflammation, and bone loss while pan-HDACi did not. In HDACi, p21 cell cycle inhibition occurs, and then TRAIL-induced apoptosis occurs. In human FLS E11 RA cells, HDACi caused upregulation of p21 and decreased proliferation and decreased IL-6 due to decreased mRNA stability. HDACi also causes reduced NF-B, decreased expression of FGF2- and IL-1 $\beta$ induced MMP-1, MMP-3, and MMP-13 in articular 
chondrocytes on TSA treatment in vitro. In macrophages of RA patients, HDACi treatment led to macrophage apoptosis through downregulation of the anti-apoptotic protein Bfl-1/A1. HDACi low doses can reduce the expression of IL-12p40 and IL-6, while high concentrations cause an increase in TNF- $\alpha$ and IL-1 $\beta$ in a mouse model with the CIA. Where low-dose Vorinostat leads to an improvement in the clinical degree of RA. 26

Table.1. Mechanism of action of HDACi12

\begin{tabular}{|c|c|c|}
\hline $\begin{array}{l}\text { Biological effects on non- } \\
\text { tumor cells }\end{array}$ & $\begin{array}{l}\text { The main effect of HDAC } \\
\text { inhibitor }\end{array}$ & Other comments \\
\hline $\begin{array}{l}\text { Regulation of different subsets of } \\
\text { immune cells }\end{array}$ & $\begin{array}{l}\text { Inhibition of dendritic cell } \\
\text { differentiation and function } \\
\text { Cytotoxicity against } \\
\text { macrophages, neutrophils, } \\
\text { and eosinophils } \\
\text { Induction of apoptosis in } \\
\text { proliferating B cells } \\
\text { Increased tumor killing by } \\
\text { NK cells and cells T cytotoxic } \\
\text { Enhanced CD8+T } \\
\text { differentiation and function } \\
\text { cellIn TReg cells, broad- } \\
\text { spectrum HDACi enhances } \\
\text { suppressor production and } \\
\text { function; HDACi class I } \\
\text { suppresses the number of } \\
\text { TReg cells. } \\
\text { Suppression of inflammatory } \\
\text { cytokine production. }\end{array}$ & $\begin{array}{l}\text { In TReg cells, broad- } \\
\text { spectrum HDACi is used for } \\
\text { inflammatory disease, } \\
\text { whereas class I HDACi is } \\
\text { used as a tumor growth } \\
\text { inhibitor. } \\
\text { HDACi is effective in } \\
\text { ameliorating arthritis } \\
\text { severity in preclinical clinics. }\end{array}$ \\
\hline Inhibition of angiogenesis & $\begin{array}{l}\text { Suppressed proangiogenic } \\
\text { gene expression }\end{array}$ & $\begin{array}{l}\text { Hypoxia induces HDAC1, } \\
\text { HDAC2 and HDAC3 }\end{array}$ \\
\hline
\end{tabular}

Expression SIRT6 expression can cause antiinflammatory in RA FLS by decreasing the expression of TNF-a, MMP and RANTES. The resveratrol activator SIRT1 has beneficial prophylactic and therapeutic effects, namely, it can cause a decrease in cartilage breakdown as well as joint protective and antiinflammatory effects. ReIA/p65 is a member of the NF$\mathrm{kB}$ family which is deacetylated by SIRT1 resulting in reduced transcriptional activity. Resveratrol reduces NF-B-mediated expression of inflammatory mediators and reduces RA symptoms. In addition, resveratrol can reduce the invasion and expression of MMP-1 and MMP-13 from RA FLS in vitro. Resveratrol in RA synovial fluid mononuclear cells plays a role in reducing the expression of monocyte chemoattractant protein-1 (MCP-1), especially with an increased lymphocyte count. Besides, resveratrol in 100 patients with RA also significantly lowered the clinical and biochemical markers such as serum C-reactive protein (CRP), TNF-a, and IL-6.27

HDACI in RA has been said to be useful in the treatment therapy that reduces the degree of severity of the disease, reduces joint swelling, reduces proinflammatory cytokines TNF-a, IL-1 $\beta$, and IL- 6 in synovial tissue or serum. In addition, there was also a decrease in cell infiltration into synovial tissue after HDACi class I and II treatment or with selective HDAC6 inhibitors. Effects of HDAC class I and II inhibitors on animal models of RA as well as from in vitro studies with human FLS RA cells and PBMCs showed good prophylactic and therapeutic results. 28

\section{Conclusion}

HDAC inhibitors in rheumatoid arthritis may reduce disease severity by reducing inflammation, swelling 
and bone and cartilage breakdown. HDACi therapy reduces cell entry into synovial joint tissue, decreases proinflammatory cytokines, and causes an increase in acetyl histone and cell cycle inhibitory regulation accompanied by an increased sensitivity to cell apoptosis.

\section{References}

1. Mellor, J. Dynamic nucleosomes and gene transcription. Trends Genet. 2006; 22: 320329.

2. Ito, K., Barnes, P. J. and Adcock, I. M. Glucocorticoid receptor recruitment of histone deacetylase 2 inhibits interleukin-1betainduced histone $\mathrm{H} 4$ acetylation on lysines 8 and 12. Mol. Cell. Biol. 2000; 20: 6891-6903.

3. Berger, S. L. The complex language of chromatin regulation during transcription. Nature. 2007; 447, 407-412

4. Kouzarides, T. Chromatin modifications and their function. Cell. 2007; 128: 693-705

5. Elimeler W, Selser C. Histone deacetylase function in CD4+ $\mathrm{T}$ cells. Nature reviews | Immunology. (C) 2018 Macmillan Publishers Limited, part of Springer Nature.

6. Wade, P. A. Transcriptional control at regulatory checkpoints by histone deacetylases: molecular connections between cancer and chromatin. Hum. Mol. Genet. 2001; 10: 693-698.

7. Strahl, B. D. and Allis, C. D. The language of covalent histone modifications. Nature (London). 2000; 403: 41-45.

8. Yoshida, M., Furumai, R., Nishiyama, M., Komatsu, Y., Nishino, N. and Horinouchi, S. Histone deacetylase as a new target for cancer chemotherapy. Cancer Chemother. Pharmacol. 2001; 48 (1): S20-S26.

9. Bassett SA, Barnett MPG. The Role of Dietary Histone Deacetylases (HDACs) Inhibitors in Health and Disease. Nutrients 2014; 6: 42734301; doi:10.3390/nu6104273.

10. Helleputte LV, Benoy V, Den Bosch LV. The Role of histone deacetylase 6 (HDAC6) in neurodegeneration. Research and Reports in Biology, 2 September 2014.

11. Forsberg, E. C. and Bresnick, E. H. Histone acetylation beyond promoters: long-range acetylation patterns in the chromatin world. Bioessays. 2001; 23: 820-830.

12. Bjerling, P., Silverstein, R. A., Thon, G., Caudy, A., Grewal, S. and Ekwall, K. Functional divergence between histone deacetylases in fission yeast by distinct cellular localization and in vivo specificity. Mol. Cell. Biol. 2002; 22: 2170-2181.

13. Falkenberg KJ, Johnstone RW. Histone deacetylases and their inhibitors in cancer, neurological diseases and immune disorders. Nature Reviews Drug Discovery | AOP, published online 18 August 2014; doi: $10.1038 / \operatorname{nrd} 4360$.

14. Barnett, M.P.G.; Bassett, S.A.; Bermingham, E.N. Epigenetics-What role could this play in functional foods and personalized nutrition? In Nutrigenomics and Nutrigenetics in Functional Foods and Personalized Nutrition; Ferguson, L.R. Ed.; CRC Press: Florence, KY, USA, 2013; 243-267.

15. Haberland, M.; Montgomery, R.L.; Olson, E.N. The many roles of histone deacetylases in development and physiology: Implications for disease and therapy. Nat. Rev. Genet. 2009; 10: $32-42$.

16. Ho, E.; Dashwood, R.H. Dietary manipulation of histone structure and function. World Rev. Nutr. Diet. 2010; 101: 95-102.

17. Kouzarides, T. Chromatin modifications and their function. Cell 2007; 128: 693-705.

18. Glozak, M.A.; Seto, E. Histone deacetylases and cancer. Oncogene 2007, 26, 5420-5432.

19. Xu, W.S.; Parmigiani, R.B.; Marks, P.A. Histone deacetylase inhibitors: Molecular mechanisms of action. Oncogene 2007; 26: 5541-5552.

20. Blander, G.; Guarente, L. The Sir2 family of protein deacetylases. Annu. Rev. Biochem. 2004; 73: 417-435. 
21. Yamamoto, H.; Schoonjans, K.; Auwerx, J. Sirtuin functions in health and disease. Mol. Endocrinol. 2007; 21: 1745-1755.

22. Shoba, B.; Lwin, Z.M.; Ling, L.S.; Bay, B.H.; Yip, G.W.; Kumar, S.D. Function of sirtuins in biological tissues. Anat. Rec. 2009; 292: 536543.

23. Simoes-Pires, C.; Zwick, V.; Nurisso, A.; Schenker, E.; Carrupt, P.-A.; Cuendet, M. HDAC6 as a target for neurodegenerative diseases: What makes it different from the other HDACs? Mol. Neurodegener. 2013.

24. Wade, P. A. Transcriptional control at regulatory checkpoints by histone deacetylases: molecular connections between cancer and chromatin. Hum. Mol. Genet. 2001; 10: 693-698.

25. Strahl, B. D. and Allis, C. D. The language of covalent histone modifications. Nature (London). 2000; 403: 41-45.
26. Finnin, M. S., Donigian, J. R., Cohen, A., Richon, V. M., Rifkind, R. A., Marks, P. A., Breslow, R. and Pavletich, N. P. Structures of a histone deacetylase homologue bound to the TSA and SAHA inhibitors. Nature (London). 1999; 401: 188-193.

27. Johnstone, R. W. Histone-deacetylase inhibitors: novel drugs for the treatment of cancer. Nat. Rev. Drug Discovery. 2002; 1, 287-299.

28. Marks, P., Rifkind, R. A., Richon, V. M., Breslow, R., Miller, T. and Kelly, W. K. Histone deacetylases and cancer: causes and therapies. Nat. Rev. Cancer. 2001; 1: 194-202. 